\title{
Experimental Study of the Two-Phase Flow Patterns of Air-Water Mixture at Vertical Bend Inlet and Outlet
}

\author{
Veyan A. Musa \\ Department of Mechanical Engineering, \\ University of Zakho, \\ Zakho, Kurdistan Region, Iraq \\ veyan.musa@staff.uoz.edu.krd
}

\author{
Lokman A. Abdulkareem \\ Department of Petroleum Engineering, \\ University of Zakho, \\ Zakho, Kurdistan Region, Iraq \\ lokman.abdulkareem@uoz.edu.krd
}

\author{
Omar M. Ali \\ Department of Mechanical Engineering, \\ University of Zakho, \\ Zakho, Kurdistan Region, Iraq \\ omar.ali@uoz.edu.krd
}

\begin{abstract}
Air-water two-phase flow in pipes introduces a noticeable challenge due to the complexity of the fluids. Thus, to estimate the best design and reasonable financing cost of the transportation pipelines where the bends are presenting a part of their accessories, the investigators should have been able to estimate the flow regime occurring at different directions. An experiment was carried out by using a $90^{\circ}$ bend fixed with two pipes where the flow was upstream from a vertical to a horizontal pipe which were representing the bend inlet and outlet respectively. Two wire-mesh sensors were used for obtaining the data of the void fractions ( $\alpha$ ) at water superficial velocities (Usl) which changed from 0.052 to $0.419 \mathrm{~m} / \mathrm{s}$, and air superficial velocities (Usg) from 0.05 to $4.7 \mathrm{~m} / \mathrm{s}$. Furthermore, the characterization of flow regimes of the air-water flow at both bend inlet and outlet were competed accurately by using void fraction analysis of the time series, Power Spectral Density (PSD), tomographic images observed by the sensor program, and the Probability Density Function (PDF) method. The flow regimes of vertical flow lines at the bend inlet were observed as bubbly, capbubble, slug, and churn flow, whereas the flow regimes of the horizontal flow line at the bend outlet were characterized as having stratified, stratified wavy, bubbly, plug, slug, wavy annular, and semi-annular flow due to the gravity and bend effects.
\end{abstract}

Keywords-flow pattern in vertical pipes; flow pattern in horizontal pipes; air-water flow; wire mesh sensor (WMS); twophase flow at bends

\section{INTRODUCTION}

Generally, two-phase flow exists in a wide scope of mechanical applications and can be noticed in many scientific fields [1]. In chemical processes various two-phase flow forms occur in pipelines, reactors, plant parts, components, and bubble columns. In each case, the two-phase flow is considered as an important factor to improve the productivity and safety of the procedures. Therefore, the suitable control of such flow properties is of significant importance to raise the efficiency of the operating system [2]. Air-water mixture flows in transportation pipelines and bends occur in a lot of applications such as the transport heat exchange pipelines, transportation water pipelines, reactor system of thermal-hydraulic, etc. This kind of flow is unpredictable because it has a complex quality while it is passed through different behavior bends when compared with single-phase flow. In fact, the two-phase flow in bends conducts as inhomogeneous stage dispersion and flow inversion occurs under the influence of gravity, buoyancy, and centrifugal forces. The primary experimental research in such studies has been presented in [3]. Authors investigated the flow distribution of air-water mixture flow through a vertical riser pipe of $7.6 \mathrm{~cm}$ diameter directed to $90^{\circ}$ bend, and they studied the impact of angular position $(\varphi)$ and phase velocity on the flow distribution. The authors submitted a straightforward model to foresee how each phase is acting along the bend, and they found the relationship between gravity and centrifugal forces which are communicated by the adjusted of Froude number and characterized by:

$$
F r=J^{2} /\left(g \times \sin \varphi \times R_{\text {curv }}\right)
$$

where $F r$ is Froude number, $J$ is the phase velocity, $R_{\text {curv }}$ is the bend curvature radius, and $\varphi$ is the angular position. At the point where $F r=1$, the phases are in harmony and have a tendency to stay in the original trajectory. When $F r<1$ the gas phase moves to the external axis side while the water phase flows in the internal axis side, and when $F r>1$, the gas tends to move to the internal axis side of the bend.

In an experimental investigation of the air-water flow patterns [4], the authors estimated the effect of $90^{\circ}$ elbow with $5.03 \mathrm{~cm}$ diameter on the bubbly flow structures in a development horizontal flow line. They used a probe conductivity dual sensor. Fifteen different conditions have been tested within the regimes of bubbly flow. Significantly, they are illustrated clearly, the elbow effect on the void fraction $(\alpha)$ distribution. In addition, the test demonstrated that the bend increases the fluctuation of the flow for both horizontal and vertical directions of the pipe cross-section area. Authors in [5] obtained similar results in a $45^{\circ}$ shape with the same diameter and conditions. In [6], an experimental study was carried out to study the effects of fluctuating forces on a $90^{\circ}, 5.25 \mathrm{~cm}$ diameter elbow. An aggregate of 36 tests was completed to cover the annular, bubbly, and slug flow regimes by using an impedance probe as measurement technique. The dominating 
force frequency was observed significantly in the slug flow pattern. The root means square values of fluctuation force persistently rise with increase in the gas flow rate and greatest value is achieved at annular flow. Authors in [7] studied experimentally the two-phase flow in a $90^{\circ}$ bend of $3.4 \mathrm{~cm}$ internal diameter. The void fractions were tested in two situations, horizontal to vertical and vertical downstream to a horizontal flow line. The test has been performed under different values of phase superficial speeds, varied from 0.21 to $0.90 \mathrm{~m} / \mathrm{s}$ for water and 0.3 to $4 \mathrm{~m} / \mathrm{s}$ for air. The experiment utilized time series PDF analysis and visual imaging technique to portray the flow conducts and patterns. The outcome where that wavy, stratified, slug, and plug regimes were noticed in the horizontal pipe In contrast, the churn and slug flow patterns were observed at the vertical pipe.

Author in [8] studied the flow regime of gas-liquid flow using PDF, liquid holdup in the domain of time series, and PSD to identify the flow regimes in the transparent inclined pipe. The experimental study in [9] exhibited the identification of air-water flow in a $50 \mathrm{~mm}$ horizontal pipe by using PDF and the void fraction of time series under different water and air superficial velocities according to the analysis estimated in [10]. They observed the following flow regimes in the horizontal pipe: annular-wavy, slug, bubbly, and stratified flow under air superficial velocities from 0.23 to $10.5 \mathrm{~m} / \mathrm{s}$ and water superficial velocities from 0.05 to $1.7 \mathrm{~m} / \mathrm{s}$ by using WMS to obtain the void fraction values. Authors in [11] identified the flow of air-water patterns in a horizontal pipe of $5 \mathrm{~cm}$ diameter by using WMS and they improved the possibility of identification flow patterns by PDF and void fraction of time series correlation technique. Many techniques have been utilized to characterize the flow patterns of the gas-liquid flow, such as X-ray tomography [2], Wire-Mesh Sensor (WMS) [12], and Electrical Capacitance Tomography (ECT). Authors in [13] obtained and used an innovated WMS sensor which depends on the conductivity alteration in the properties between flow fluids. The WMS was exhibited in 1998 at tomographic imaging, and after it has been improved in [14] as an optimistic technique to obtain the hold-up $(\mathrm{H})$ of water in oil depending on the conductivity of the liquid. Finally, the comprehension of the air-water flow properties accounts on the knowledge of the flow patterns occurring inside the pipes, therefore the exact prediction of the two-phase flow pattern is the starting point for designing systems able to save energy and diminish system collapse by calculating the void fractions and estimating the bend effect on the air-water flow regimes of the vertical riser pipe to the horizontal flow line.

\section{EXPERIMENTAL DESIGN}

The current investigation was conducted at the Research Center of Helmholtz Centrum Dresden Rossendorf (HZDR) in Germany. The flow consisted of air and tap water at room temperature of $22^{\circ} \mathrm{C}$ with atmospheric pressure. A $90^{\circ}$ bend of a $15.35 \mathrm{~cm}$ curvature radius has been joined between the riser vertical pipe and the horizontal pipe with a diameter of $6.7 \mathrm{~cm}$. Two wire mesh sensors were fixed on the flow path before and after the bend ends with a $20 \mathrm{~cm}$ distance from the bend ends. The flow direction was vertically upstream to the horizontal flow line. The void fractions $(\alpha)$ of time series information were obtained by two sensors (WMS1 and WMS2) as shown in Figure 1. The flow patterns of the flow lines were examined for 13 different values of air superficial velocity (Usg) ranging from 0.05 to $4.7 \mathrm{~m} / \mathrm{s}$ and constant water superficial velocity (Usl) ranging from 0.052 to $0.419 \mathrm{~m} / \mathrm{s}$.
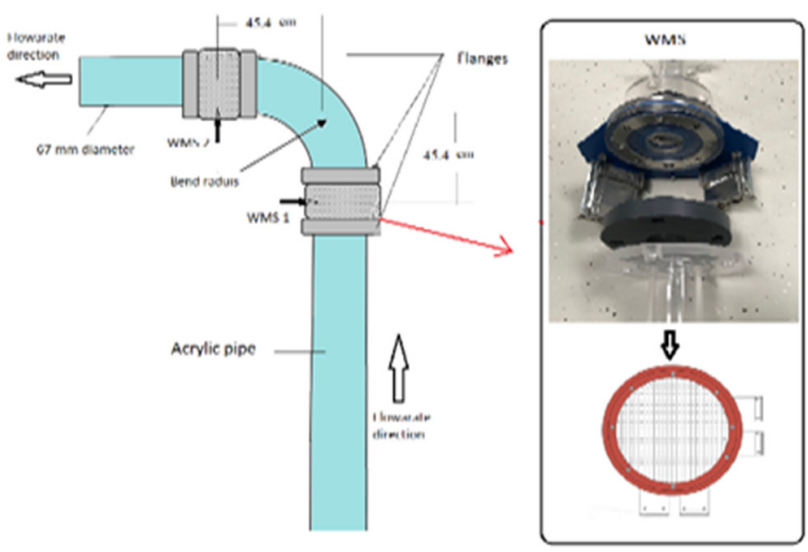

Fig. 1. The $90^{\circ}$ bend frame.

III. RESULTS

The following methods were utilized to classify the flow regimes occurring at the bend inlet in the vertical pipe and at the bend outlet in the horizontal pipe: the time series of void fraction, the tomographic images of the WMS program, Power Spectral Density (PSD), Probability Density Function (PDF), and mean void fraction and liquid holdup $(\mathrm{H})$.

\section{A. At Steady Liquid Superficial Velocity (Usl) of $0.052 \mathrm{~m} / \mathrm{s}$}

At Usl $=0.052 \mathrm{~m} / \mathrm{s}$ and gas superficial velocity $U_{s}=0.05 \mathrm{~m} / \mathrm{s}$ at the bend inlet, the mean void fraction fluctuates at 0.1 with a few peaks (Figure 2(a)). The plot of PDF exhibits a single peak of high PDF value of 0.17 with a void fraction $\alpha=0.1$ (Figure 2(b)). The amplitude value of the spectrum accomplishes its maximum value at $1.5 \mathrm{~Hz}$ and declines gradually to the right (Figure 2(c)). The plot features are referred to the bubbly pattern. At the bend outlet, the plot of the void fraction is shown as a straight line with some waves under the mean void fraction of about 0.5 . The PDF curve shows a hill shape with $\alpha$ values between 0.3 to 0.6 , whereas the plot of PSD is characterized by a peak value of about 70 near zero frequency. Regarding the amount of air and water that entered the bend, the outlet stratified wavy regime is conducted normally because the turbulent flow which occurred at the bend outlet was waving the phases. By increasing the Usg value to $0.28 \mathrm{~m} / \mathrm{s}$ at the bend inlet, the mean void fraction fluctuated frequently under the values of 0.2 and 0.7 (Figure 3(a)) The PDF plot exhibits two peaks at PDF values 0.01 and 0.05 (Figure 3(b)), while the PSD plot shows a single peak with a maximum of about 60 at $1.75 \mathrm{~Hz}$ (Figure 3(c)). This value is declined and fluctuated with increase in frequency to the right and is characterized as slug flow. The increment in Usg led to an increase in the Taylor bubbles length and shrinking of the air bubbles into the water, thus, the slug flow was obtained. At the bend outlet for the horizontal flow line, the flow was observed 
as stratified wavy, which is estimated by the plot of the void fraction as a straight line at 0.7 with a few waves. The PDF plot is characterized by a single peak at 0.1 , and the PSD plot shows a maximum spectrum peak of about 80 near $0 \mathrm{~Hz}$. The waves in the flow tend to increase the PSD value, thus the stratified wavy plot depicts a higher value than the stratified flow plot. The stratified wavy pattern is obtained as a result of the bend effect, where the small amount of water is mixed with considerably high values of Usg.

When the Usg value reached $1.4 \mathrm{~m} / \mathrm{s}$, the flow pattern was of a churn regime at the bend inlet for the vertical flow line. The mean void fraction oscillates under a high value of about 0.8 (Figure 4(a)). The PDF plot has one peak at 0.75 (Figure 4(b)), while the maximum spectrum peak shows a small value of 30 at $2 \mathrm{~Hz}$ with a larger base scope (Figure 4(c)). The increase of Usg value raises the instability of the water slugs. When the Usg achieves a critical point, the water slugs will be penetrated and this prompts the flow pattern to change its form into churn flow. At the bend outlet for the horizontal flow line, the flow regime is observed as stratified wavy because the increment of Usg with a little amount of water mass flow rate make flow wavy and unstable due to the bend influence.

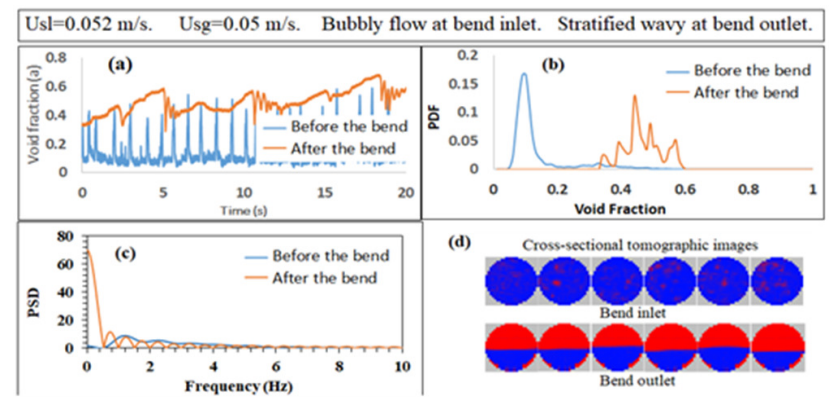

Fig. 2. Flow pattern identification methods by (a) void fraction of time series, (b) PDF technique, (c) PSD analysis, and (d) tomographic images at $\mathrm{Usl}=0.052 \mathrm{~m} / \mathrm{s}$ and $\mathrm{Usg}=0.05 \mathrm{~m} / \mathrm{s}$.

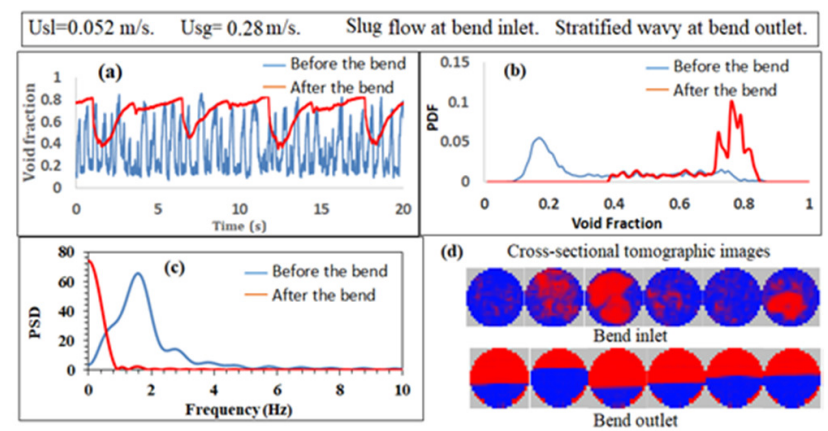

Fig. 3. Flow pattern identification methods by using (a) void fraction of time series, (b) PDF technique, (c) PSD analysis, and (d) tomographic images at $U \mathrm{sl}=0.052 \mathrm{~m} / \mathrm{s}$ and $\mathrm{Usg}=0.28 \mathrm{~m} / \mathrm{s}$.

At Usg $=2.36 \mathrm{~m} / \mathrm{s}$, the flow regime is identified as churn flow at the bend inlet as displayed in Figure 5 while the wavy annular flow at the bend outlet is characterized through the PDF chart by small peaks fluctuating around 0.15 with $(\alpha)$ tailing from 0.7 to 0.95 (Figure 5(b)). The PSD curve displays the wavy annular pattern showing $10 \%$ less power spectral than the plot of the stratified wavy regime (Figure 5 (c)). The increment of Usg with the differentiator of the density between the two phases, in addition to the effect of the bend forces the water phase to flow at the pipe base with waves.

When increasing the Usg value to $4.7 \mathrm{~m} / \mathrm{s}$ as illustrated in Figure 6, semi-annular flow is observed at the bend outlet in comparison with the churn flow from the vertical flow line at the bend inlet. At the bend outlet, the mean void fraction conducts as a straight line with some waves at the high value of 0.9 (Figure 6(a)) and the PDF plot has a single peak shifted to the right (Figure 6(b)), referring to semi-annular flow. The interfaces occurring between air and water at the bend pushed the little amount of water to flow along with the pipe basement and air flew on it with high velocity.

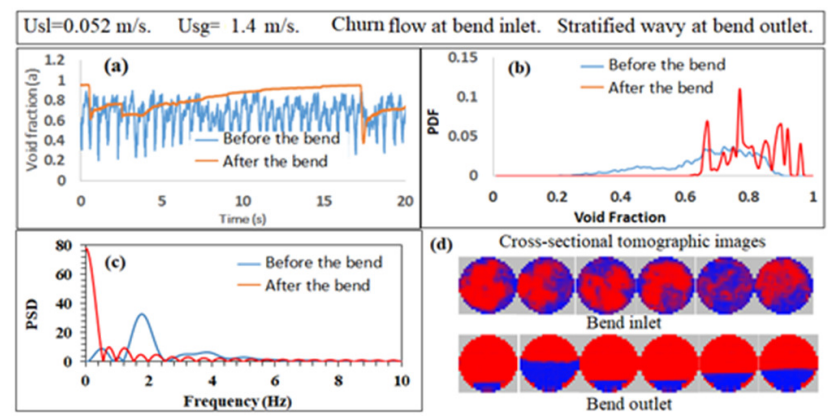

Fig. 4. Flow pattern identification methods by using (a) void fraction of time series, (b) PDF technique, (c) PSD analysis, and (d) tomographic images at $U s \mathrm{l}=0.052 \mathrm{~m} / \mathrm{s}$ and $U s g=1.4 \mathrm{~m} / \mathrm{s}$.

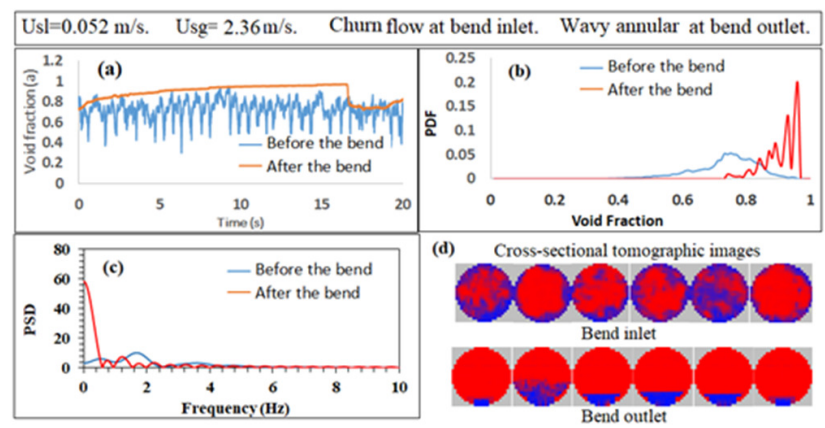

Fig. 5. Flow pattern identification methods by using (a) void fraction of time series, (b) PDF technique, (c) PSD analysis, and (d) tomographic images at $U s \mathrm{l}=0.052 \mathrm{~m} / \mathrm{s}$ and $U s g=2.36 \mathrm{~m} / \mathrm{s}$.

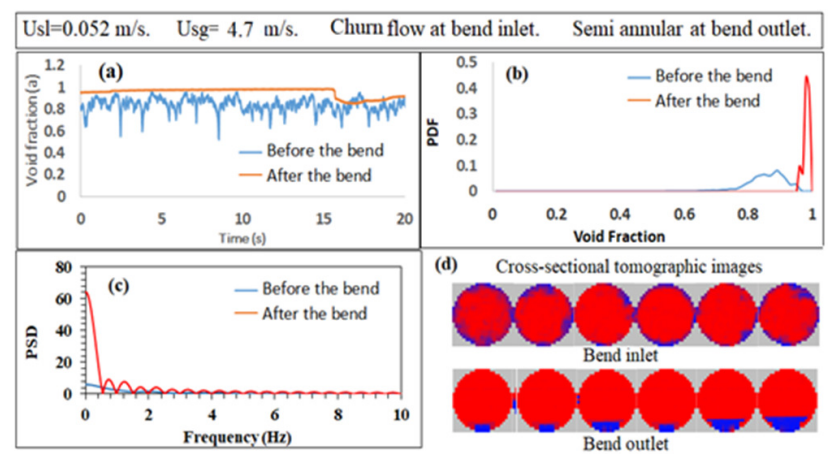

Fig. 6. Flow pattern identification methods by using (a) void fraction of time series, (b) PDF technique, (c) PSD analysis, and (d) tomographic images at $U s \mathrm{l}=0.052 \mathrm{~m} / \mathrm{s}$ and $U s g=4.7 \mathrm{~m} / \mathrm{s}$. 


\section{B. At Steady Liquid Superficial Velocity Usl $=0.262 \mathrm{~m} / \mathrm{s}$}

For the horizontal flow line (the bend outlet) at Usg $=0.05 \mathrm{~m} / \mathrm{s}$ as in Figure 7, the bubbly flow is estimated when the mean void fraction is fluctuated at 0.1 with a few peaks at 0.3 (Figure 7(a)). The bubbly flow is also characterized through the PDF chart: the plot shows a single peak to the left with a small base from 0.1 to 0.2 (Figure 7(b)), whereas the power spectrum shows a maximum value of 10 at $0.75 \mathrm{~Hz}$ (Figure $7(\mathrm{c})$ ). The amount of water was enough for the pipe diameter to fill the cross-section area in spite of bend impacts, thus, the bubbly flow at the vertical pipe was observed to have the same pattern of the horizontal flow line. When the Usg reached $0.28 \mathrm{~m} / \mathrm{s}$ (Figure 8 ), the flow pattern was similar to plug flow at the bend outlet for the horizontal flow line in contrast with the slug flow that was observed at the vertical flow line. The plug flow is characterized by the time series method when the mean void fraction fluctuates at very low values near to zero and high void fraction of 0.7 at the same time (Figure 8(a)). The PDF curve shows two peaks at 0.13 and 0.05 at void fractions 0.1 and 0.6 respectively (Figure $8(\mathrm{~b})$ ). Furthermore, the power spectrum exhibits high value of about 350 near $0 \mathrm{~Hz}$ (Figure 8(c)).

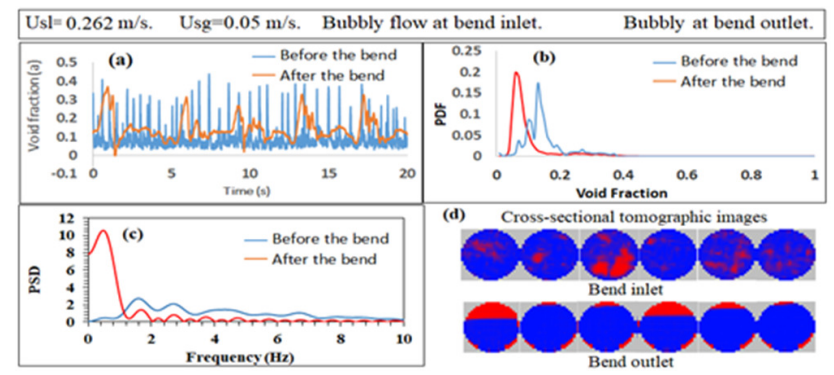

Fig. 7. Flow pattern identification methods by using (a) void fraction of time series, (b) PDF technique, (c) PSD analysis, and (d) tomographic images at $U s \mathrm{sl}=0.262 \mathrm{~m} / \mathrm{s}$ and $U s g=0.05 \mathrm{~m} / \mathrm{s}$.

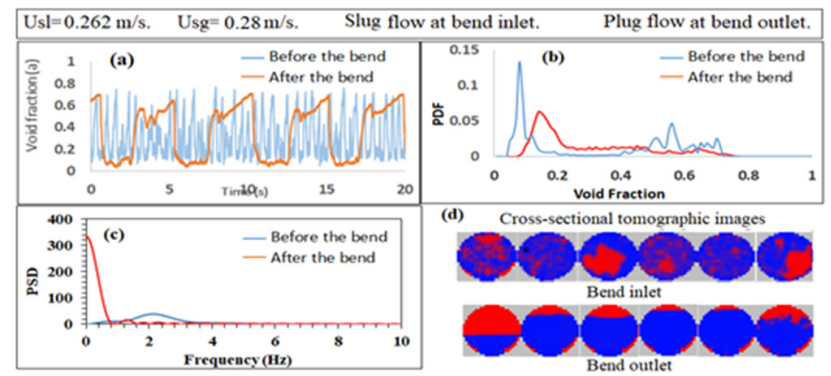

Fig. 8. Flow pattern identification methods by using (a) void fraction of time series, (b) PDF technique, (c) PSD analysis, and (d) tomographic images at $\mathrm{Usl}=.262 \mathrm{~m} / \mathrm{s}$ and $\mathrm{Usg}=0.28=\mathrm{m} / \mathrm{s}$.

Slug flow is observed at Usg values from 0.34 to $0.9 \mathrm{~m} / \mathrm{s}$ at the bend outlet for the horizontal flow line comparing with the slug flow that arrived from the bend inlet as showed in Figure 9. For the horizontal flow line, as Figure 9(a) shows, the slug pattern is identified when the void fraction fluctuates at high values (when the water height is less than the pipe radius) and has low values near zero (when the horizontal cross-sectional area is filled with water). The PDF curve shows two or more peaks around 0.08 at low and high void fraction values (Figure 9(b)). Moreover, the power spectrum of the slug flow exhibited a higher value of 450 at very low frequencies (Figure 9(c)). The water slugs which arrived from the vertical pipe due to the higher void fraction and moderate Usg values ranged from 0.34 to $0.9 \mathrm{~m} / \mathrm{s}$ are reverse inside the bend center flow line due to the gravity force that led the water to move at the bottom pipe base with considerable water slugs flow frequently at the top.

Stratified wavy flow is observed at Usg increases from 1.4 to $2.36 \mathrm{~m} / \mathrm{s}$ as showed in Figure 10, and the wavy annular regime is recognized at $U s g=4.7 \mathrm{~m} / \mathrm{s}$ as demonstrated in Figure 11. When the Usg increases more, to $2.83 \mathrm{~m} / \mathrm{s}$, it breaks the slugs of water down due to the high velocity of the flow that produces wavy annular flow which is noticed at the bend outlet of the horizontal flow line.

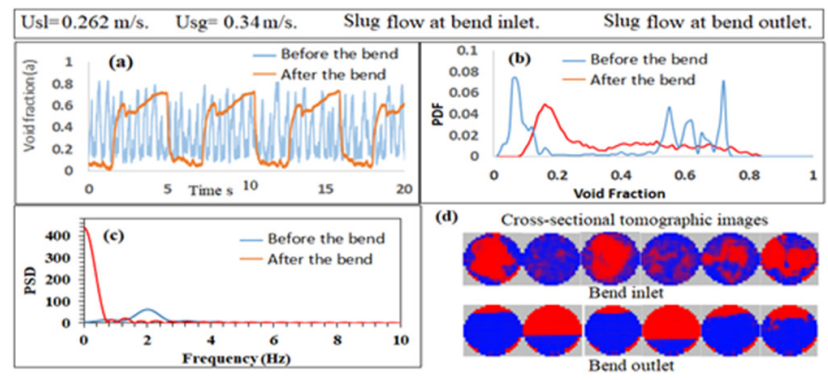

Fig. 9. Flow pattern identification methods by using (a) void fraction of time series, (b) PDF technique, (c) PSD analysis, and (d) tomographic images at $\mathrm{Usl}=0.262 \mathrm{~m} / \mathrm{s}$ and $\mathrm{Usg}=0.34 \mathrm{~m} / \mathrm{s}$.

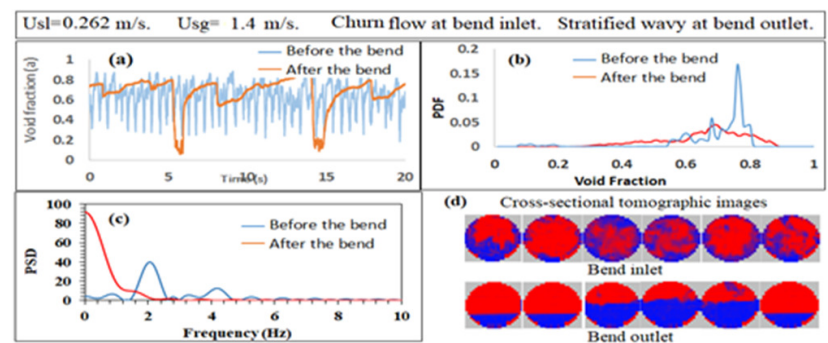

Fig. 10. Flow pattern identification methods by using (a) void fraction of time series, (b) PDF technique, (c) PSD analysis, and (d) tomographic images at $U s \mathrm{~s}=0.262 \mathrm{~m} / \mathrm{s}$ and $U s g=1.4 \mathrm{~m} / \mathrm{s}$.

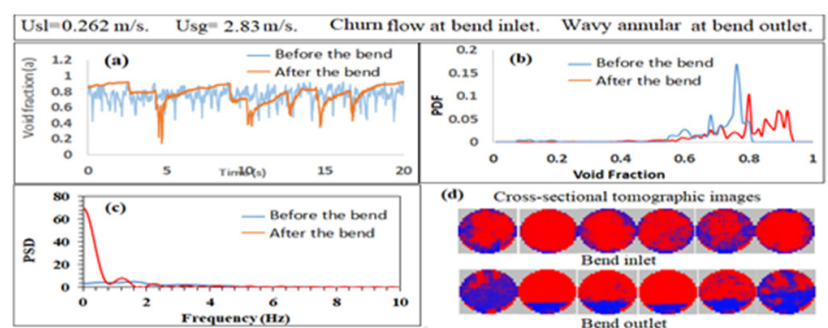

Fig. 11. Flow pattern identification methods by using (a) void fraction of time series, (b) PDF technique, (c) PSD analysis, and (d) tomographic images at $U s \mathrm{~s}=0.262 \mathrm{~m} / \mathrm{s}$ and $\mathrm{Usg}=2.83 \mathrm{~m} / \mathrm{s}$.

\section{Mean Void Fraction and Liquid Holdup $(H)$}

The liquid holdup $(\mathrm{H})$ of the two-phase flow is characterized as a liquid volumetric rate fraction over the total mixture volumetric rates. The mean liquid holdup $(\mathrm{H})$ values 
are obtained by calculating the average of all local crosssectional liquid holdup values over the time series. The mean $\mathrm{H}$ of the air-water flow diminishes linearly when the air superficial velocity, Usg, gradually increases at steady water superficial velocity Usl. This might clarify that bubbles expand steeply with increasing Usg rates [15]. Additionally, it is observed that the increasing or decreasing of the rate of the liquid phase is affected directly by the $(\mathrm{H})$, and this influence is noticeable. Figure 12 shows the plots of the mean $(\mathrm{H})$ for constant Usl values of $0.052,0.157,0.262,0.314$, and $0.419 \mathrm{~m} / \mathrm{s}$ regarding Usg values altered from 0.05 to $4.7 \mathrm{~m} / \mathrm{s}$ at the vertical and undeveloped horizontal flow lines. It appears that the mean liquid holdup $\mathrm{H}$ diminishes steeply by the increasing of Usg at all conditions in both flow lines (b) and (c). The mean liquid holdup at low Usl diminishes dramatically at the bend outlet as compared with the bend inlet. The correlations between the mean liquid holdups at different Usg values of this test are compared with [7-9].

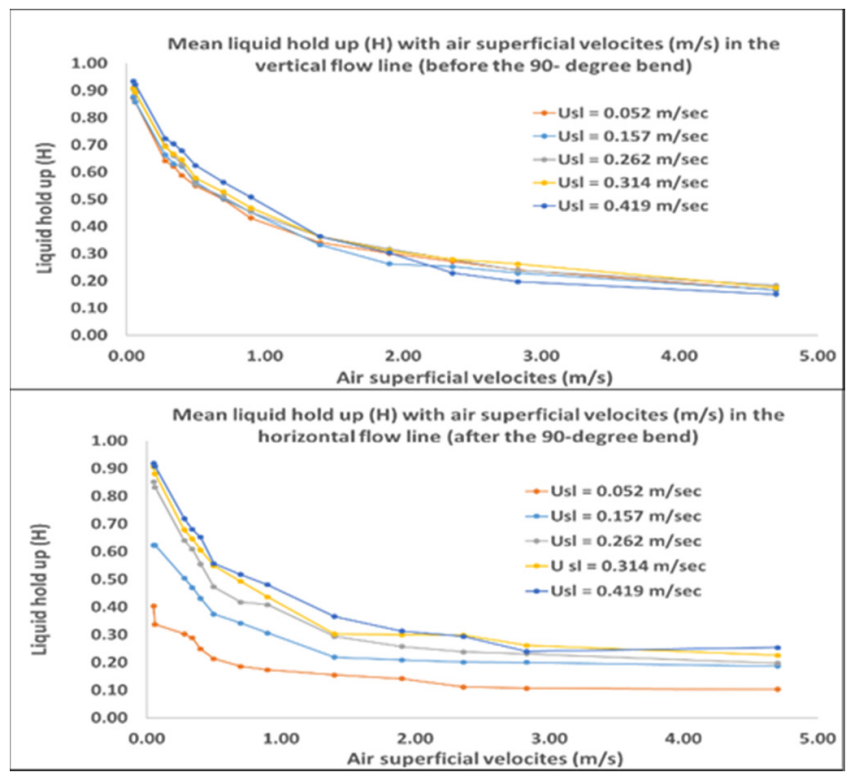

Fig. 12. Liquid holdup $(\mathrm{H})$ of the Usg for vertical and horizontal flow line.

\section{CONCLUSION}

The increasing of water superficial velocity did not change the pattern in the vertical pipe, but it has a clear impact due to the effect of the 90-degree bend, gravitational force, and the buoyancy force on the flow patterns of the horizontal flow lines. These forces acted on the flow and forced the water to move inside the region of the bend centerline while the air flowed at the outside centerline of the bend. As a result, the large bubbles were broken up in the bend entrances during the imbalance which occurred by the surface tension and the centrifugal force. The flow patterns at the bend outlet may show a different behavior which depends on the bend curvature radius and diameter, fluid properties, temperature, etc. Consequently, the bubbly flow regime in the vertical pipe changed to stratified-wavy flow at low Usl values. In addition, the bubbly flow pattern in the vertical pipe altered to bubbly and plug flow regime at higher values of Usl in the horizontal pipe. Besides, the slug flow in the vertical pipe changed to the stratified-wavy pattern at low Usl values, and to slug pattern as the value of Usl increased. The churn pattern in the vertical pipe varied to the stratified-wavy, and semi-annular pattern at low Usl in the horizontal flow lines and, to wavy-annular flow pattern at higher Usl. Finally, no stratified flow regime was observed at the horizontal flow lines under the tests, and no bubbly, plug, and slug flow was observed at the horizontal flow lines due to the low values of Usl.

\section{REFERENCES}

[1] C. T. Crowe, Multiphase Flow Handbook, CRC Press, 2005

[2] M. J. Da Silva, Impedance Sensors for Fast Multiphase Flow Measurement and Imaging, $\mathrm{PhD}$ Thesis, Technische Universität Dresden, 2008

[3] G. C. Gardner, P. H. Neller, "Phase distributions in flow of an air-water mixture round bends and past obstructions at the wall of a 76- $\mathrm{mm}$ bore tube", Proceedings of the Institution of Mechanical Engineers, Vol. 184, No. 33, pp. 93-101, 1969

[4] S. Kim, J. H. Park, G. Kojasoy, J. M. Kelly, "Local interfacial structures in horizontal bubbly flow with 90-degree bend", 14th International Conference on Nuclear Engineering, July 17-20, 2006

[5] J. D. Talley, S. Kim, T. Guo, G. Kojasoy, "Geometric effects of 45-deg elbow in horizontal air-water bubbly flow", Nuclear Technology, Vol. 167, No. 1, pp. 2-12, 2009

[6] Y. Liu, M. Shuichiro, H. Takashi, I. Mamoru, M. Hideyuki, K. Yoshiyuki, K. Koichi, "Experimental study of internal two-phase flow induced fluctuating force on a $90^{\circ}$ elbow", Chemical Engineering Science, Vol. 76, No. 2012, pp. 173-187, 2012

[7] F. Saidj, R. Kibboua, A. Azzi, N. Ababou, B. J. Azzopardi, "Experimental investigation of air-water two-phase flow through vertical $90^{\circ}$ bend", Experimental Thermal and Fluid Science, Vol. 57, pp. 226 234,2014

[8] L. A. Abdulkareem, Tomographic Investigation of Gas-Oil Flow in Inclined Risers, PhD Thesis, University of Nottingham, 2011

[9] M. De Salve, G. Monni, B. Panella, "Horizontal air-water flow analysis with wire mesh sensor", in: 6th European Thermal Sciences Conference (Eurotherm 2012), IOP Publishing, 2012

[10] A. E. Dukler, M. G. Hubbard, "A Model for gas-liquid slug flow in horizontal and near horizontal tubes", Industrial \& Engineering Chemistry Fundamentals, Vol. 14, No. 4, pp. 337-347, 1975

[11] W. Liu, C. Tan, F. Dong, "Local characteristic of horizontal air-water two-phase flow by wire-mesh sensor", Transactions of the Institute of Measurement and Control, Vol. 40, No. 3, pp. 746-761, 2016

[12] H. F. Velasco Pena, O. M. H. Rodriguez, "Applications of wire-mesh sensors in multiphase flows", Flow Measurement and Instrumentation, Vol. 45, pp. 255-273, 2015

[13] H. M. Prasser, A. Bottger, J. Zschau, "A new electrode-mesh tomography for gas-liquid flows", Flow Measurement and Instrumentation, Vol. 9, No. 2, pp. 111-119, 1998

[14] I. D. Johnson, Method and Apparatus for Measuring Water in Crude Oil, United States Patent 4644263, 1987

[15] D. J. Nicklin, J. O. Wilkes, J. F. Davidson, "Two-phase flow in vertical tubes", Proceedings of the Institution of Mechanical Engineers, Vol. 40, No. 1, pp. 61-68, 1962 